\title{
Efficacy of Epidermal Growth Factor Receptor Tyrosine Kinase Inhibitors in the Adjuvant Setting for Patients with Resected Epidermal Growth Factor Receptor Mutant Non-Small Cell Lung Cancer: A Meta-Analysis with 11 Trials
}

\author{
Qifan Yin $^{a}$ Xuejiao Xun ${ }^{b}$ Guang Yang ${ }^{a}$ Hongshang Cui Huining Liu ${ }^{a}$ \\ ${ }^{a}$ Department of Thoracic Surgery, Hebei General Hospital, Shijiazhuang, PR China; ${ }^{b}$ Department of Pharmacy, \\ Hebei General Hospital, Shijiazhuang, PR China
}

\section{Keywords}

Epidermal growth factor receptor tyrosine kinase inhibitors · Adjuvant setting · Non-small cell lung cancer . EGFR mutation $\cdot$ Meta-analysis

\begin{abstract}
Background: Epidermal growth factor receptor tyrosine kinase inhibitors (EGFR-TKIs) have recently become the standard first-line therapy for advanced non-small cell lung cancer (NSCLC) patients harboring EGFR mutations. This study aimed to define the role of EGFR-TKI treatment in the adjuvant setting of patients with resected EGFR-mutant NSCLC. Methods: Three online databases (PubMed, Embase, and the Cochrane Library) were used to conduct systematic research to search for studies published before June 1, 2020. The disease-free survival (DFS) and overall survival (OS) of patients with resected EGFR-mutant NSCLC after radical surgery treated with EGFR-TKIs versus non-EGFR-TKIs in the adjuvant setting were compared. Based on rigorous self-defined inclusion and exclusion criteria, studies were selected, and a meta-analysis was performed using hazard rate (HR) and 95\% Cls as effective measures. Results: Eleven studies, published between 2011 and 2020, with a total of 1,900 patients, were included in this meta-analysis. EGFR-TKI treatment showed a significant beneficial effect on DFS (HR 0.42; 95\% $\mathrm{Cl} 0.31-0.57)$ and OS (HR 0.62; 95\% Cl 0.45-0.86) for patients with resected EGFR-mutant NSCLC after radical resection in the adjuvant setting. Conclusion: Our meta-analysis results
\end{abstract}

suggested that EGFR-TKI treatment improved the DFS and OS of completely resected patients with EGFR-mutant NSCLC compared with non-EGFR-TKI treatment in the adjuvant setting. In the future, our conclusion should be confirmed by additional large-scale and well-designed clinical trials.

두 2021 S. Karger AG, Basel

\section{Introduction}

Lung cancer is considered as the main cause of cancer-related mortality worldwide, and non-small cell lung cancer (NSCLC) comprises approximately $85 \%$ of all lung carcinoma patients [1]. Radical resection, which provides the highest probability of cure, is the most effective treatment for early and some locally advanced NSCLC [2]. However, despite optimal surgical management, the 5-year survival rate of these patients remains suboptimal, ranging between 25 and $73 \%$ for pathological stages I and IIIA [3]; therefore, adjuvant therapy after surgery is crucial. Furthermore, the results from large randomized trials and meta-analyses revealed that cisplatin-based chemotherapy as adjuvant therapy is strongly recommended for stage II-IIIA NSCLC after complete resection [4-6], which gives rise to a survival improvement of $5 \%$ at 5 years (from 40 to $45 \%$ ) [7]. However, we cannot neglect the adverse effects of cytotoxic agents, which are the primary reason for refusal of or insufficient chemotherapy [5]. Therefore, although 
adjuvant chemotherapy might confer a survival benefit to some patients with stage II-IIIA NSCLC, we should seek further survival improvements through the use of alternative treatments with better tolerability than adjuvant chemotherapy.

Epidermal growth factor receptor tyrosine kinase inhibitors (EGFR-TKIs) have been the standard first-line therapy for patients with advanced EGFR-mutant NSCLC; these confer better disease-free survival (DFS) and overall survival (OS) than cisplatin-based chemotherapy $[8,9]$. Cisplatin-based chemotherapy is the recommended standard-of-care adjuvant treatment for stage IIa-IIIb resected NSCLC, irrespective of EGFR mutation status $[3,10]$. In the past 5 years, several trials that compared the efficacy and toxicity of EGFR-TKIs with those of chemotherapy or placebo among resected patients harboring EGFR mutations were published. Some trials indicated that EGFR-TKIs are superior to chemotherapy as an initial treatment for patients with EGFRmutant NSCLC following surgical resection [11-15], whereas other trials showed no difference $[16,17]$. The multicenter single-arm SELECT trial, performed by investigators from the Memorial Sloan Kettering Cancer Center, demonstrated the feasibility of adjuvant erlotinib in patients with EGFR-mutant NSCLC and revealed that EGFR-TKIs could improve the survival of EGFR-mutant patients after surgery compared with historical controls [18]. However, the previous trials BR19 [16] and RADIANT [17], without inclusion of EGFR mutation status in the patient selection criteria, yielded negative outcomes. In clinical practice, whether EGFR-TKIs in adjuvant treatment for patients with resected EGFR-mutant NSCLC improve survival compared with adjuvant chemotherapy or placebo is still controversial.

We conducted this meta-analysis of EGFR-mutant NSCLC patients after radical surgery treated with EGFRTKIs versus non-EGFR-TKIs in the adjuvant setting to define the role of EGFR-TKI treatment in the adjuvant setting for patients with resected EGFR-mutant NSCLC.

\section{Materials and Methods}

\section{Search Strategy}

All studies comparing survival with EGFR-TKI treatment with survival with non-EGFR-TKI treatment in the adjuvant setting in completely resected patients with early-stage NSCLC were eligible for inclusion. To identify eligible studies, we searched PubMed, Embase, and the Cochrane Library without any restrictions regarding publication status, type, date, or language. The search was updated to June 1, 2020. The main search terms included "adjuvant" or "postoperative"; "epidermal growth factor receptor tyrosine kinase inhibitors," "EGFR-TKI," "gefitinib," "erlotinib," "icotinib," "dacomitinib," or "osimertinib"; and "non small cell lung cancer," "NSCLC," "lung neoplasms," or "adenocarcinoma of lung." We also reviewed the reference list for relevant articles.

\section{Study Selection}

Studies that met the following criteria were included: (1) adult patients with pathologically confirmed NSCLC; (2) studied patients underwent primary radical resection harboring EGFR mutations (exon 19 deletion or exon 21 Leu858Arg); (3) evaluation of the efficacy of adjuvant EGFR-TKIs versus chemotherapy, placebo; adjuvant EGFR-TKIs combined with chemotherapy versus chemotherapy alone; (4) reporting at least one relevant clinical endpoint event, for instance, DFS or OS with long-term follow-up; and (5) containing original data sufficient to calculate HR or $p$ values. All the enrolled studies were published in English without any restriction on publication type.

The exclusion criteria included (1) abstracts, letters, case reports, reviews, duplicates, or nonclinical studies; (2) studied patients who had previously undergone neoadjuvant therapy (neoadjuvant chemotherapy or neoadjuvant chemoradiotherapy) or had previously been exposed to EGFR-TKIs or immunologic therapy before surgery and those with incomplete resection; (3) singlearm studies reporting adjuvant EGFR-TKI outcomes; (4) studies with irretrievable or insufficient data for statistical analysis; and (5) original articles with unavailable full text.

Some studies analyzed the effects of EGFR inhibitors combined with conventional systematic therapy (EGFR-TKIs + chemotherapy) compared with those without EGFR inhibitors (only conventional systematic therapy), whereas others employed EGFR-TKIs instead of chemotherapy (EGFR-TKIs vs. chemotherapy) after complete resection in patients with EGFR-mutant NSCLC. Therefore, to evaluate the effects on patients who had received EGFRTKIs in the adjuvant treatment setting compared with those who had not received these, we included all studies in our meta-analysis.

\section{Data Extraction and Quality Assessment}

We extracted all the corresponding information including the first author, publication year, region, number of participants, EGFR mutation rate, median EGFR-TKI treatment duration, median follow-up, participants' characteristics (age, stage, and treatment strategy), and outcomes from each selected study. Two independent investigators carried out the work of study selection and data extraction. If disagreement emerged, a consensus was reached after discussion with a third author.

The methodological quality of the enrolled articles was evaluated independently by the two investigators. A rating system called the Newcastle-Ottawa Scale [19], which consists of 3 aspects, i.e., selection ( $0-4$ points), comparability ( $0-2$ points), and outcome (0-3 points), was used for nonrandomized cohort studies. The total score achieved from the scores of the 3 sections ranged from 0 to 9 , which was positively correlated with the study's quality. Furthermore, studies awarded with scores $>5$ were considered to be of acceptable quality. The Cochrane Collaboration's tool for estimating the risk of bias (5.3.0) was used to evaluate the quality of the included randomized controlled trials (RCTs) [20] with the following methodological items: random sequence generation, allocation concealment, blinding of participants and personnel, blinding of outcome assessment, incomplete outcome data, selective reporting, and other potential sources of bias. Each item was classified as low risk, high risk, or unclear risk, determining the general quality when taken together. The disagreements between the two investigators were resolved by discussion with a third investigator.

\section{Statistical Analysis}

Review Manager 5.3 and STATA 12.0 software was applied to conduct all statistical analyses. Based on the methods of Tierney et al. [21] and Parmar et al. [22], we directly extracted HR and 95\% CIs from each study or estimated these data. An HR $<1$ showed that EGFR-TKI treatment in the adjuvant setting might provide 
Fig. 1. Flow diagram of study selection.

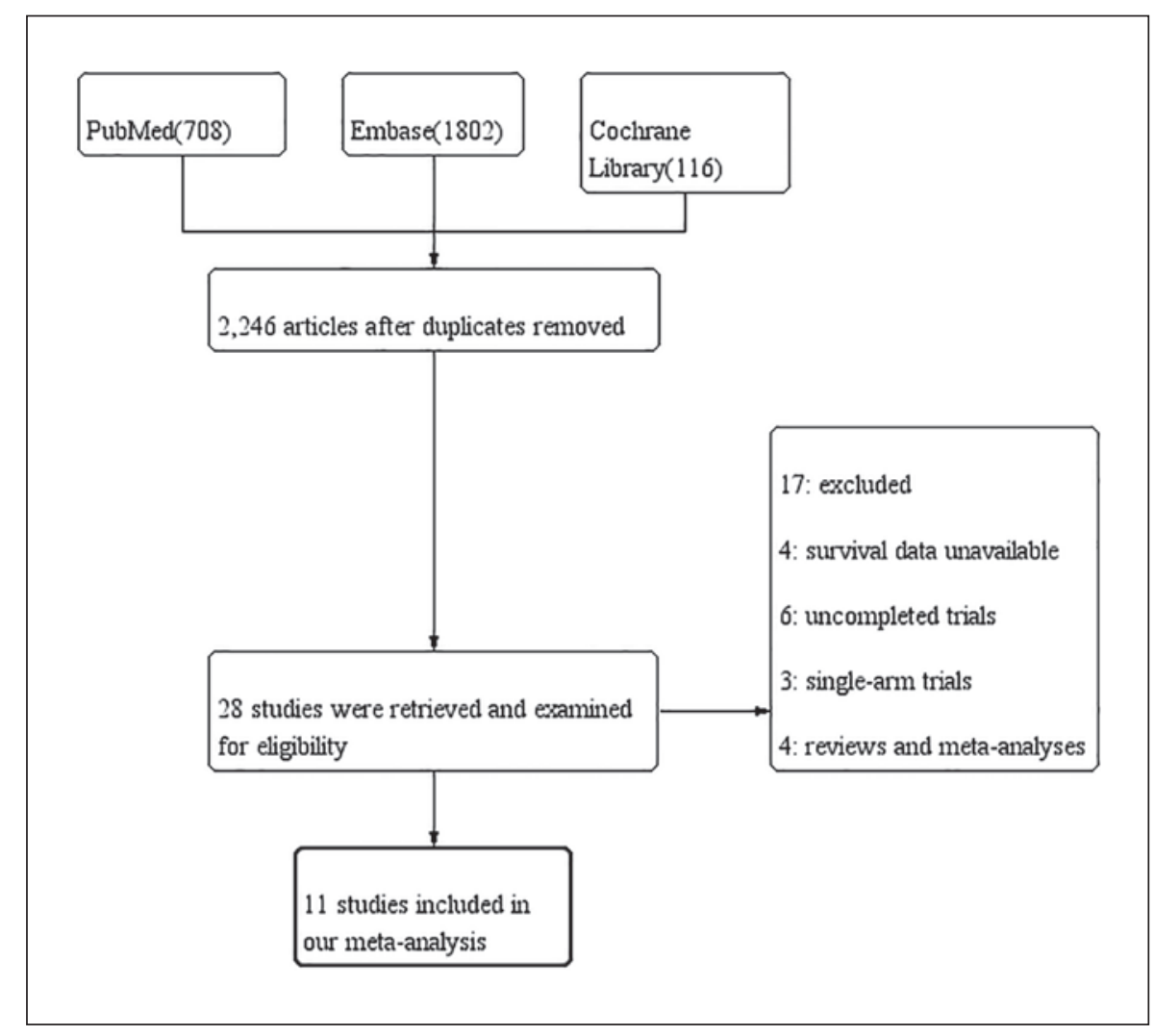

survival benefits to patients with resected EGFR-mutant NSCLC. $I^{2}$ tests were conducted to test for heterogeneity, and no heterogeneity was assumed if $p>0.1$ or $I^{2}<50 \%$ using a fixed-effects statistical model, whereas $p<0.1$ or $I^{2}>50 \%$ was suggestive of substantial journal pre-proof heterogeneity, so a random-effects model was used. Publication bias was estimated visually by funnel plots and statistically by Egger's and Begg's tests. A $p$ value $<0.05$ was deemed statistically significant.

\section{Results}

\section{Study Characteristics}

In the initial search, a total of 2,246 articles were identified by combining the results from PubMed (708), Embase $(1,802)$, and the Cochrane Library (116) and removing duplicates (Fig. 1). After reviewing the titles and abstracts of these articles, the full texts of 28 studies were retrieved and examined for eligibility, 6 of which were excluded because they were not completed, 4 trials were excluded because of unavailable survival data, 3 studies were excluded due to a single-arm design, and 4 studies were excluded because they were reviews and meta-analyses. The remaining 11 studies of acceptable quality were finally included in our meta-analysis according to the inclusion and exclusion criteria, 8 of which were RCTs and 3 were retrospective comparative studies. Figure 1 presents the detailed study selection procedure in the form of a flow diagram. In 9 studies, all of the patients were diagnosed with EGFR-mutant NSCLC [11-14, 2326]. Moreover, the other 2 studies (BR19 and RADIANT) $[16,17]$ reported detailed data for EGFR-mutant subgroups, though the proportions were relatively low ( 4 and $16.5 \%$, respectively). We enrolled only a portion of patients with EGFR mutations in the BR19 and RADIANT trials into the meta-analysis to compare OS and DFS because some of those patients did not harbor EGFR mutations in the study. A total of 1,900 patients were enrolled in the pooled analysis, including 887 in the EGFRTKI treatment group and 1,013 in the non-EGFR-TKI treatment group in the adjuvant setting. All patients enrolled in this meta-analysis harbored EGFR-sensitive mutations. Table 1 shows the characteristics of all the 11 included studies.

\section{Effect of EGFR-TKI Treatment in the Adjuvant} Setting on the DFS of Patients with EGFR-Mutant NSCLC

Eleven studies (1,900 patients) were included in the analysis of DFS for the comparison between the EGFRTKI group (TKIs only/TKIs plus chemotherapy) and the non-EGFR-TKI group (placebo/chemotherapy) in the adjuvant setting for patients with resected EGFR-mutant NSCLC, considering the heterogeneity $\left(I^{2}=62.2 \%, p=\right.$ 0.003). Therefore, a random-effects model was applied. 


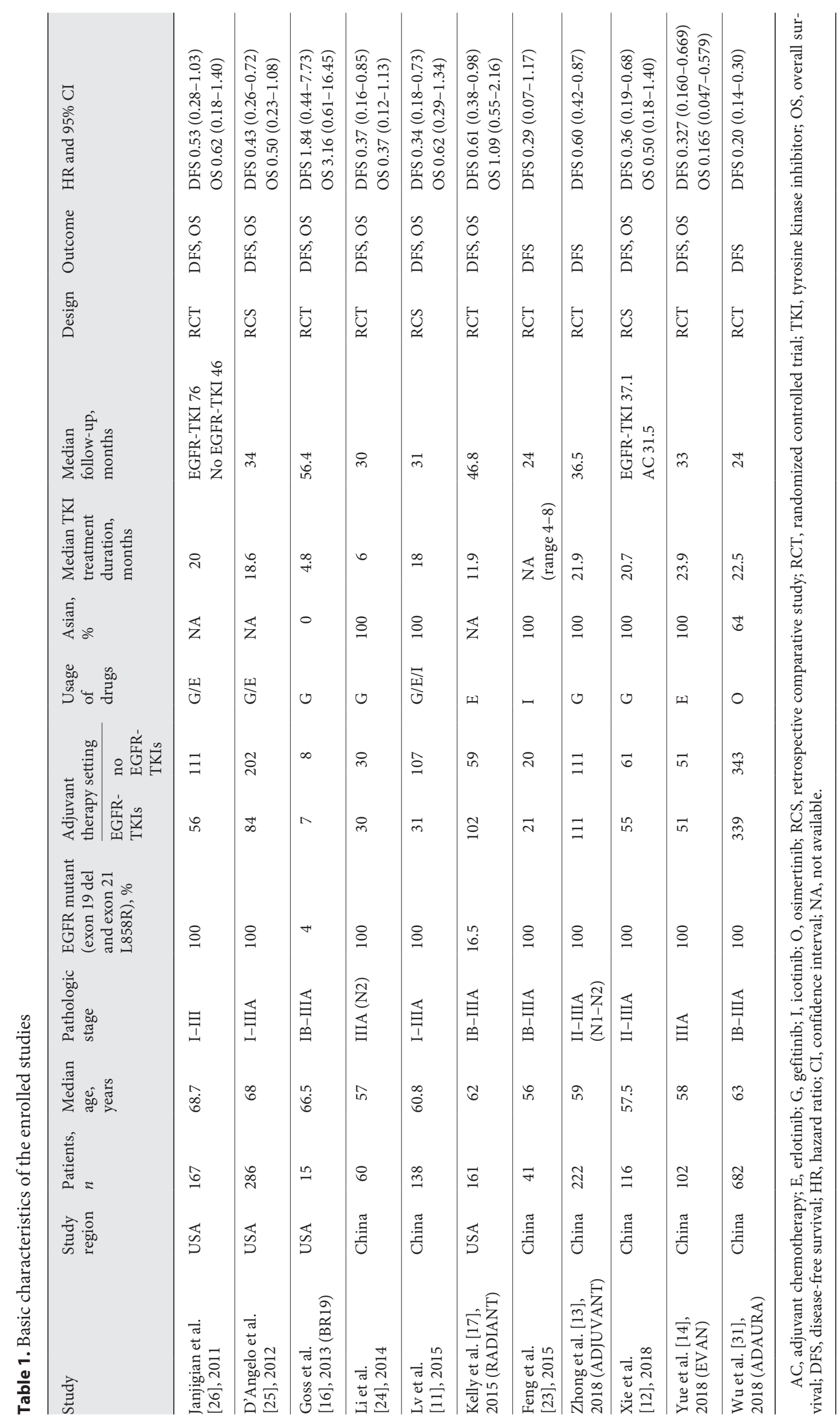




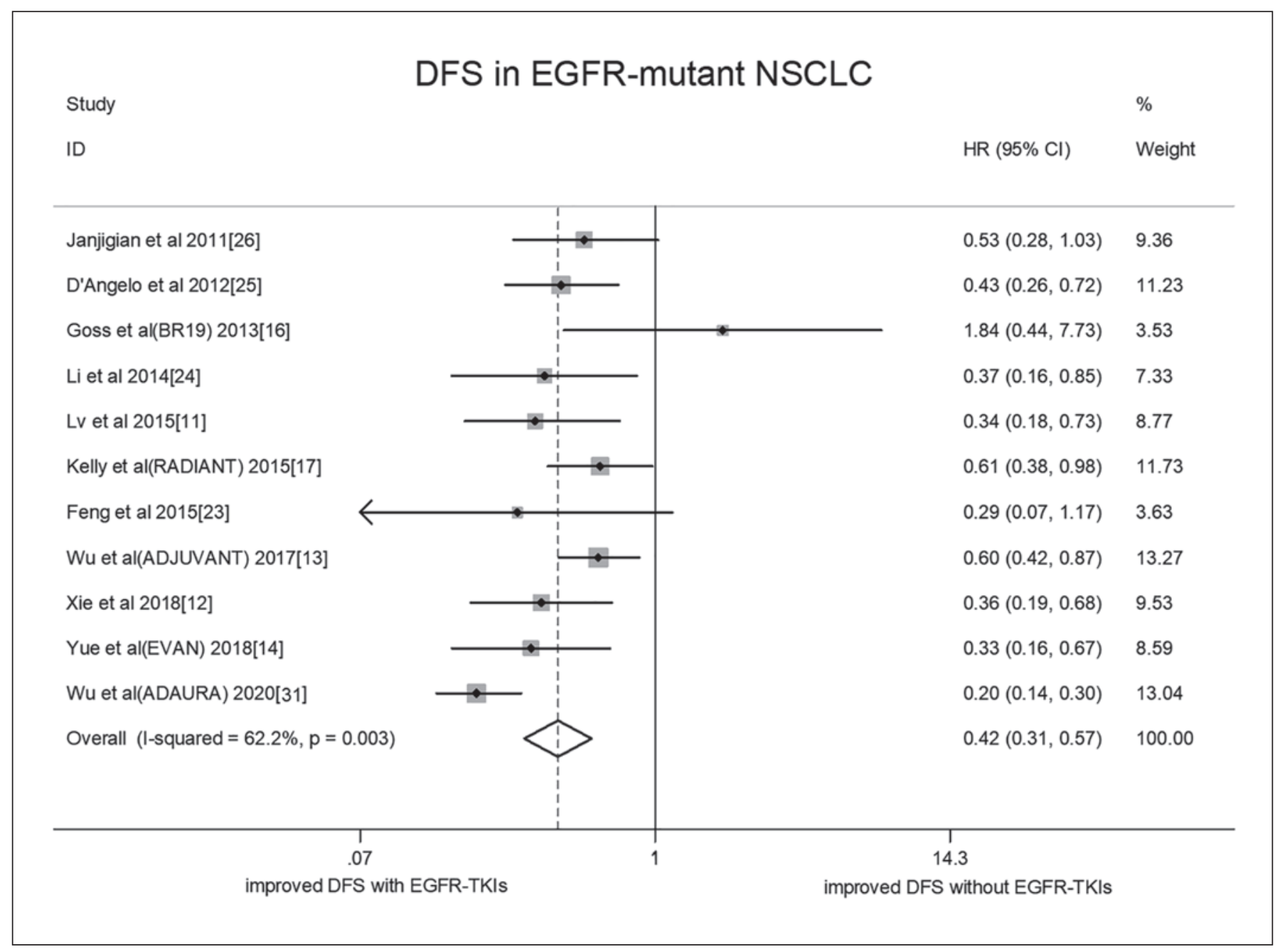

Fig. 2. Forest plots of the HR of disease-free survival (DFS) with epidermal growth factor receptor tyrosine kinase inhibitors (EGFR-TKIs) versus non-EGFR-TKIs in the adjuvant setting in patients with EGFR-mutant non-small cell lung cancer (NSCLC) after radical resection.

Our results clearly demonstrated that EGFR-TKIs showed a significant beneficial effect on DFS in patients with EGFR-mutant NSCLC (HR 0.42; 95\% CI 0.31-0.57; Fig. 2).

\section{Effect of EGFR-TKI Treatment in the Adjuvant}

Setting on the OS of Patients with EGFR-Mutant NSCLC

A meta-analysis of 8 studies (955 patients) showed effects of EGFR-TKIs versus non-EGFR-TKIs in the adjuvant setting on the OS of patients with EGFR-mutant NSCLC after radical resection. EGFR-TKI treatment exhibited superiority over non-EGFR-TKI therapy in the adjuvant setting (HR 0.62; 95\% CI 0.45-0.86; heterogeneity $I^{2}=41.3 \%, p=0.103$; Fig. 3 ).

\section{Publication Bias}

Based on the data for DFS and OS, publication bias was evaluated by Begg's funnel plot and Egger's linear regres- sion test. In our meta-analysis, no publication bias was found in the DFS group ( $p=0.325$ [>0.05] for Begg's test) and in the OS group ( $p=0.262[>0.05]$ for Begg's test) (Fig. 4).

\section{Discussion}

According to the previous clinical trials and metaanalyses, cisplatin-based chemotherapy as the previous standard adjuvant treatment does improve the OS time for NSCLC patients, regardless of the EGFR mutation status, after radical operation [4-6]. However, its benefits have reached a plateau, and a high risk of recurrence and adverse events also limits its application. In recent years, EGFR-TKIs have been the standard first-line therapy for patients with EGFR-mutant advanced NSCLC. However, whether they can be employed as adjuvant therapy for 


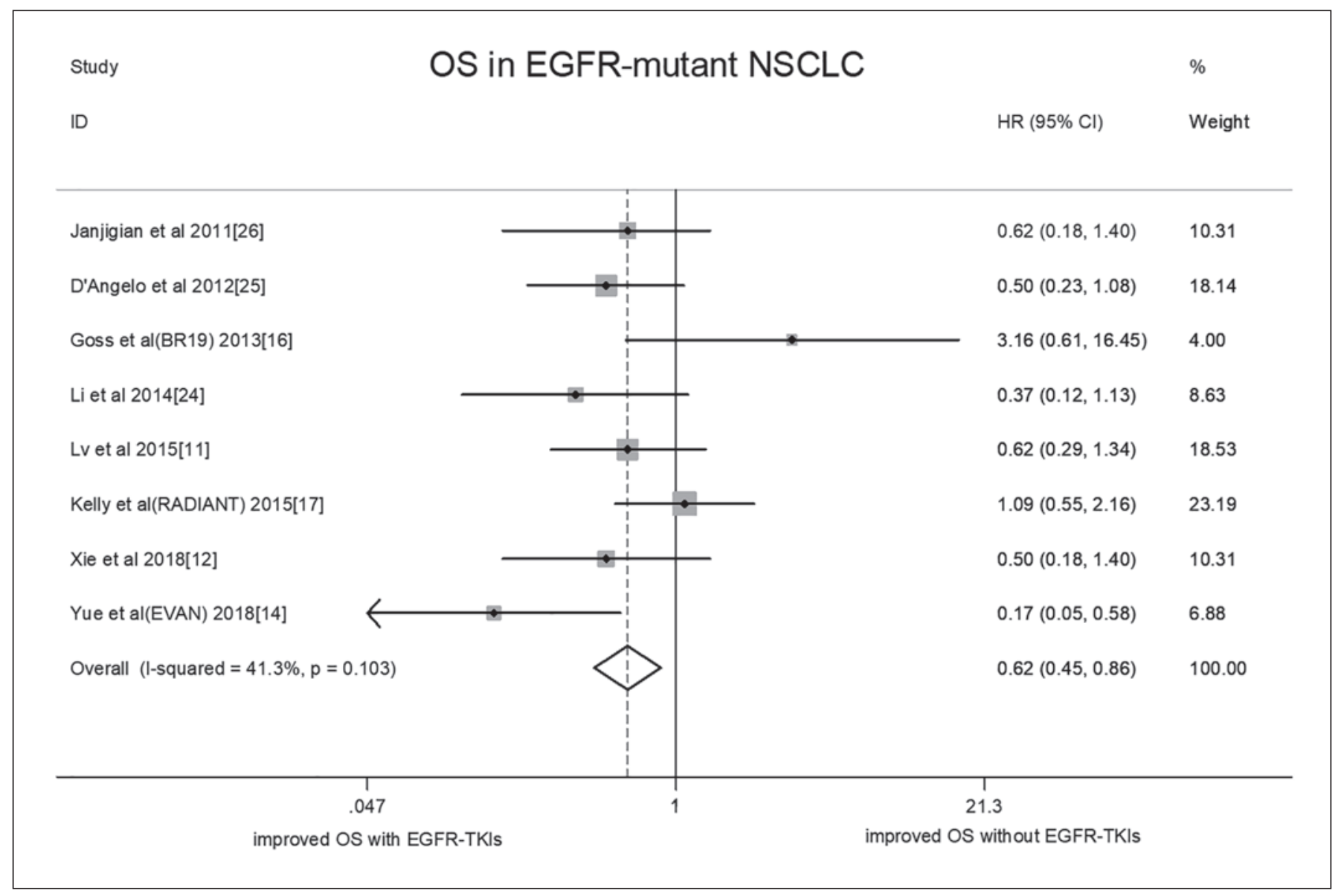

Fig. 3. Forest plots of the HR of overall survival (OS) with epidermal growth factor receptor tyrosine kinase inhibitors (EGFR-TKIs) versus non-EGFR-TKIs in the adjuvant setting in patients with EGFR-mutant non-small cell lung cancer (NSCLC) after radical resection.

those patients with resected EGFR-mutant NSCLC is still controversial. Hence, our research came about to investigate these factors.

Our meta-analysis showed that EGFR-TKI treatment for EGFR-mutant NSCLC patients improved the DFS and OS compared with non-EGFR-TKI treatment in the adjuvant setting after radical resection. Two previous meta-analyses showed that EGFR-mutant NSCLC patients benefited from adjuvant TKI treatment after radical resection $[27,28]$. However, our updated meta-analysis included 2 large clinical trials (EVAN and ADJUVANT research). These studies therefore strengthened the evidence for the efficacy of adjuvant EGFR-TKI treatment in terms of DFS and OS in patients with EGFR-mutant NSCLC. Furthermore, our meta-analysis results notably suggested that patients with EGFR-mutant NSCLC may benefit from EGFR-TKI treatment in the adjuvant setting in terms of DFS and OS after complete surgical resection.

The single-arm phase II SELECT study revealed that patients with EGFR-mutant stage IB-IIIA resected NSCLC might benefit from adjuvant EGFR-TKIs [18]. Pa- tients $(n=100)$ with resected stage IA-IIIA (the 7th edition of the American Joint Committee on Cancer Staging System) EGFR-mutant NSCLC were treated with erlotinib ( $150 \mathrm{mg} /$ day for 2 years) after standard adjuvant chemotherapy, with or without radiotherapy. The median follow-up was 5.2 years, and the 2-year DFS rate was $88 \%$ (96\% with stage I, 78\% with stage II, and $91 \%$ with stage III). The median DFS and OS have not been reached, and the 5-year DFS and OS rates were 56\% (95\% CI 45-66) and $86 \%$ (95\% CI 77-92). A retrospective study reported a trend toward an improvement in DFS among individuals with resected stage I-III lung adenocarcinomas harboring EGFR mutations compared with a historical comparator [26]. These 2 studies presented a low rate of recurrence and an encouraging OS with adjuvant TKIs. The limitation of these studies was that they were not RCTs.

Moreover, EGFR-TKIs have no effect on patients with wild-type EGFR. In unselected patients with advanced NSCLC, EGFR-TKIs did not improve survival compared with a placebo [29]. For unselected patients with resected lung cancer, the treatment with adjuvant EGFR-TKIs did 
Fig. 4. a Publication bias for disease-free survival between epidermal growth factor receptor tyrosine kinase inhibitors (EGFRTKIs) and non-EGFR-TKIs in the adjuvant setting. b Publication bias for overall survival between EGFR-TKIs and non-EGFRTKIs in the adjuvant setting.
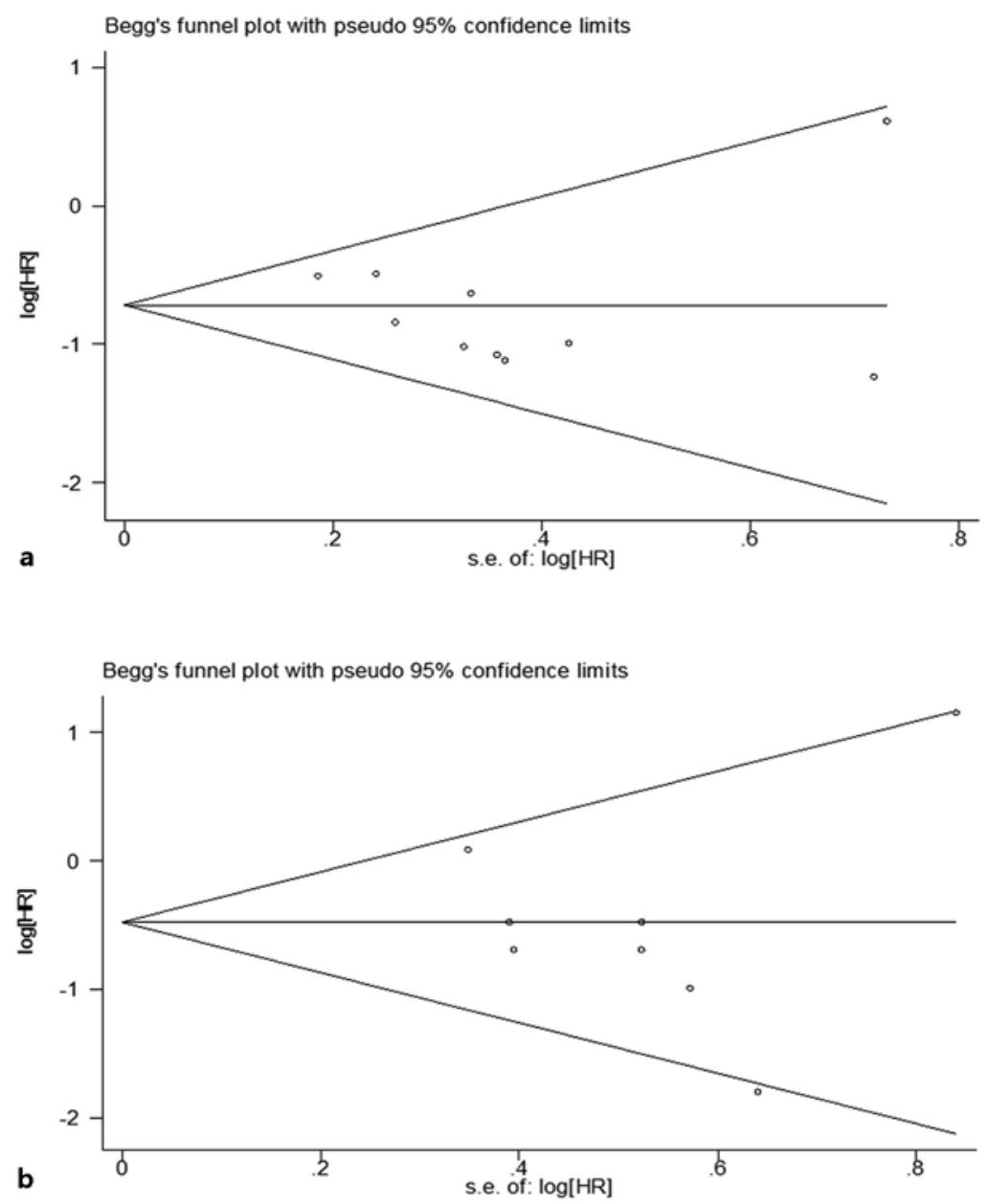

not prolong survival. The BR19 [16] and RADIANT trials [17] were two large RCTs that compared adjuvant EGFRTKIs with placebo in unselected and resected NSCLC patients. The BR19 trial was a negative trial. It recruited unselected patients; only 15 (4\%) of them had EGFR mutations. Most of these patients had wild-type EGFR. Therefore, the BR19 study was underpowered because it was terminated prematurely. The RADIANT trial also included unselected patients. Among the 973 enrolled patients, 161 (16.5\%) were in the EGFR mutation subgroup, wherein erlotinib prolonged the DFS of the patients. The patients with EGFR mutations in the BR19 and RADIANT trials were recruited and included in our meta-analysis.

Osimertinib is a third-generation EGFR-TKI that effectively and selectively inhibits EGFR-sensitive mutations and EGFR-resistant mutations (T790M). In a phase III trial of patients with EGFR-mutant advanced NSCLC, the first-line osimertinib treatment compared with gefitinib or erlotinib resulted in clinically and significantly improved progression-free survival (median 18.9 vs. 10.2 months; HR 0.46; 95\% CI 0.37-0.57; $p<0.001)$. Osimertinib is superior to the standard first-generation EGFRTKIs such as gefitinib and erlotinib as the first-line treatment strategy for EGFR-mutant advanced NSCLC [30]. The ADAURA trial was a phase III, double-blind, randomized study of osimertinib versus placebo in earlystage NSCLC patients who harbored EGFR-sensitive mutations after complete surgical resection. This study also confirmed that adjuvant osimertinib improved the DFS for EGFR-mutant early-stage NSCLC after radical resection. However, the data on OS from the ADAURA trial were premature [31].

The safety and tolerability have to be taken into account when comparing EGFR-TKI treatment with nonEGFR-TKI treatment in the adjuvant setting for resected NSCLC patients with EGFR mutations. For the EGFRTKI group, the most frequent adverse events were rash, liver dysfunction, and diarrhea [32], and the most common adverse events associated with adjuvant chemotherapy treatment were hematologic toxic effects and appetite 
loss [33]. The patients who were treated with adjuvant EGFR-TKIs experienced fewer grade 3-4 adverse events than those treated with adjuvant chemotherapy, and EGFR-TKIs have also been shown to cause no increase in toxicity even when combined with chemotherapy or radiotherapy [34]. All of the results clearly support the idea that EGFR-TKIs do not increase grade 3-4 adverse events and may be a promising treatment option for patients with resected EGFR-mutant NSCLC compared with nonEGFR-TKIs in the adjuvant setting.

Although our meta-analysis showed that EGFR-TKI treatment improved the DFS and OS compared with nonEGFR-TKI treatment in the adjuvant setting for patients with resected EGFR-mutant NSCLC, there are still many questions that need to be addressed regarding EGFR-TKI treatment for patients with EGFR-mutant NSCLC after radical resection, for example, which stage of lung cancer benefits most from adjuvant EGFR-TKIs? What is the optimal duration of adjuvant EGFR-TKI treatment? Which is the most optimal treatment regimen, adjuvant EGFRTKI alone or in combination with adjuvant chemotherapy, radiotherapy, or VEGF inhibitors? We need more well-designed clinical trials to answer this question.

There are some limitations to our meta-analysis which should be addressed. First, we included several retrospective studies, increasing the heterogeneity of this metaanalysis. Second, the median duration of EGFR-TKI treatment differed in each study, which could cause some selection bias. Besides, the BR19 trial might contribute to some publication bias due to its limited sample size.

\section{Conclusions}

Adjuvant therapies are required to prevent disease recurrence and improve patient survival after surgery. Our meta-analysis results suggest that EGFR-TKI treatment improved the DFS and OS of patients with completely resected EGFR-mutant NSCLC compared with non-EGFR-TKI treatment in the adjuvant setting. In the future, our conclusions should be confirmed by additional largescale and well-designed clinical trials.

\section{Statement of Ethics}

This study conforms to the ethical guidelines in a priori approval by the local Ethics Committee of Hebei General Hospital. Our research was approved on May 20, 2020, by the Ethics Committee of Hebei General Hospital. All enrolled patients signed informed consent.

\section{Conflict of Interest Statement}

The authors declare no conflicts of interest.

\section{Funding Sources}

No funding was involved in this study.

\section{Author Contributions}

H.L. conceived the study. G.Y. collected the data. X.X. analyzed the data and performed the statistical analyses. Q.Y. drafted the manuscript. H.C. provided important intellectual contributions and critically revised the manuscript. All authors read and approved the final manuscript.

\section{Data Availability Statement}

The data sets used and analyzed during the current study are available from the corresponding author on reasonable request.

\section{References}

1 Ettinger DS, Wood DE, Aisner DL, Akerley W, Bauman J, Chirieac LR, et al. Non-Small Cell Lung Cancer, Version 5.2017, NCCN Clinical Practice Guidelines in Oncology. J Natl Compr Canc Netw. 2017 Apr;15(4):50435.

2 Steger V, Walles T, Kosan B, Walker T, Kyriss T, Veit S, et al. Trimodal therapy for histologically proven N2/3 non-small cell lung cancer: mid-term results and indicators for survival. Ann Thorac Surg. 2009 Jun;87(6): 1676-83.

3 Vansteenkiste J, Crinò L, Dooms C, Douillard JY, Faivre-Finn C, Lim E, et al. 2nd ESMO Consensus Conference on Lung Cancer: early-stage non-small-cell lung cancer consensus on diagnosis, treatment and follow-up. Ann Oncol. 2014 Aug;25(8):1462-74.
4 Winton T, Livingston R, Johnson D, Rigas J, Johnston M, Butts C, et al.; National Cancer Institute of Canada Clinical Trials Group; National Cancer Institute of the United States Intergroup JBR.10 Trial Investigators. Vinorelbine plus cisplatin vs. observation in resected non-small-cell lung cancer. N Engl J Med. 2005 Jun;352(25):2589-97.

5 Pignon JP, Tribodet H, Scagliotti GV, Douillard JY, Shepherd FA, Stephens RJ, et al.; LACE Collaborative Group. Lung adjuvant cisplatin evaluation: a pooled analysis by the LACE Collaborative Group. J Clin Oncol. 2008 Jul;26(21):3552-9.

6 Arriagada R, Auperin A, Burdett S, Higgins JP, Johnson DH, Le Chevalier T, et al.; NSCLC Meta-analyses Collaborative Group. Adjuvant chemotherapy, with or without postop- erative radiotherapy, in operable non-smallcell lung cancer: two meta-analyses of individual patient data. Lancet. 2010 Apr; 375(9722):1267-77.

7 NSCLC Meta-Analysis Collaborative Group. Preoperative chemotherapy for non-small-cell lung cancer: a systematic review and meta-analysis of individual participant data. Lancet. 2014 May;383(9928): 1561-71.

8 Zhou C, Wu YL, Chen G, Feng J, Liu XQ, Wang $\mathrm{C}$, et al. Final overall survival results from a randomised, phase III study of erlotinib versus chemotherapy as first-line treatment of EGFR mutation-positive advanced non-small-cell lung cancer (OPTIMAL, CTONG-0802). Ann Oncol. 2015 Sep;26(9): 1877-83. 
9 Sugawara S, Oizumi S, Minato K, Harada T, Inoue A, Fujita $\mathrm{Y}$, et al. Randomized phase II study of concurrent versus sequential alternating gefitinib and chemotherapy in previously untreated non-small cell lung cancer with sensitive EGFR mutations: NEJ005/ TCOG0902. Ann Oncol.2015 May;26(5):88894.

10 Kris MG, Gaspar LE, Chaft JE, Kennedy EB, Azzoli CG, Ellis PM, et al. Adjuvant Systemic Therapy and Adjuvant Radiation Therapy for Stage I to IIIA Completely Resected NonSmall-Cell Lung Cancers: American Society of Clinical Oncology/Cancer Care Ontario Clinical Practice Guideline Update. J Clin Oncol. 2017 Sep;35(25):2960-74.

11 Lv C, An C, Feng Q, Ma Y, Li S, Wang J, et al. A Retrospective Study of Stage I to IIIa Lung Adenocarcinoma after Resection: What Is the Optimal Adjuvant Modality for Patients with an EGFR Mutation? Clin Lung Cancer. 2015 Nov;16(6):e173-81.

12 Xie H, Wang H, Xu L, Li M, Peng Y, Cai X, et al. Gefitinib versus Adjuvant Chemotherapy in Patients with Stage II-IIIA Non-Small-Cell Lung Cancer Harboring Positive EGFR Mutations: A Single-Center Retrospective Study. Clin Lung Cancer. 2018 Nov;19(6):484-92.

13 Zhong WZ, Wang Q, Mao WM, Xu ST, Wu L, Shen Y, et al.; ADJUVANT investigators. Gefitinib versus vinorelbine plus cisplatin as adjuvant treatment for stage II-IIIA (N1-N2) EGFR-mutant NSCLC (ADJUVANT/ CTONG1104): a randomised, open-label, phase 3 study. Lancet Oncol. 2018 Jan;19(1): 139-48.

14 Yue D, Xu S, Wang Q, Li X, Shen Y, Zhao H, et al. Erlotinib versus vinorelbine plus cisplatin as adjuvant therapy in Chinese patients with stage IIIA EGFR mutation-positive nonsmall-cell lung cancer (EVAN): a randomised, open-label, phase 2 trial. Lancet Respir Med. 2018 Nov;6(11):863-73.

15 Tsuboi M, Kato H, Nagai K, Tsuchiya R, Wada H, Tada $\mathrm{H}$, et al. Gefitinib in the adjuvant setting: safety results from a phase III study in patients with completely resected non-small cell lung cancer. Anticancer Drugs. 2005 Nov;16(10):1123-8.

16 Goss GD, O’Callaghan C, Lorimer I, Tsao MS, Masters GA, Jett J, et al. Gefitinib versus placebo in completely resected non-small-cell lung cancer: results of the NCIC CTG BR19 study. J Clin Oncol. 2013 Sep;31(27):3320-6.
17 Kelly K, Altorki NK, Eberhardt WEE, O’Brien MER, Spigel DR, Crinò L, et al. Adjuvant Erlotinib Versus Placebo in Patients With Stage IB-IIIA Non-Small-Cell Lung Cancer (RADIANT): A Randomized, Double-Blind, Phase III Trial. J Clin Oncol. 2015 Dec;33(34):400714

18 Pennell NA, Neal JW, Chaft JE, Azzoli CG, Jänne PA, Govindan R, et al. SELECT: A Phase II Trial of Adjuvant Erlotinib in $\mathrm{Pa}$ tients with Resected Epidermal Growth Factor Receptor-Mutant Non-Small-Cell Lung Cancer. J Clin Oncol. 2019 Jan;37(2):97-104.

19 Stang A. Critical evaluation of the NewcastleOttawa scale for the assessment of the quality of nonrandomized studies in meta-analyses. Eur J Epidemiol. 2010 Sep;25(9):603-5.

20 Michaelis R, Tang V, Wagner JL, Modi AC, LaFrance WC Jr, Goldstein LH, et al. Cochrane systematic review and meta-analysis of the impact of psychological treatments for people with epilepsy on health-related quality of life. Epilepsia. 2018 Feb;59(2):315-32.

21 Tierney JF, Stewart LA, Ghersi D, Burdett S, Sydes MR. Practical methods for incorporating summary time-to-event data into metaanalysis. Trials. 2007 Jun;8(1):16.

22 Parmar MK, Torri V, Stewart L. Extracting summary statistics to perform meta-analyses of the published literature for survival endpoints. Stat Med. 1998 Dec;17(24):2815-34.

23 Feng S, Wang Y, Cai K, Wu H, Xiong G, Wang $\mathrm{H}$, et al. Randomized Adjuvant Chemotherapy of EGFR-Mutated Non-Small Cell Lung Cancer Patients with or without Icotinib Consolidation Therapy. PLoS One. 2015 Oct; 10(10):e0140794.

24 Li N, Ou W, Ye X, Sun HB, Zhang L, Fang Q, et al. Pemetrexed-carboplatin adjuvant chemotherapy with or without gefitinib in resected stage IIIA-N2 non-small cell lung cancer harbouring EGFR mutations: a randomized, phase II study. Ann Surg Oncol. 2014 Jun; 21(6):2091-6.

25 D’Angelo SP, Janjigian YY, Ahye N, Riely GJ, Chaft JE, Sima CS, et al. Distinct clinical course of EGFR-mutant resected lung cancers: results of testing of 1118 surgical specimens and effects of adjuvant gefitinib and erlotinib. J Thorac Oncol. 2012 Dec;7(12):181522.
26 Janjigian YY, Park BJ, Zakowski MF, Ladanyi M, Pao W, D’Angelo SP, et al. Impact on disease-free survival of adjuvant erlotinib or gefitinib in patients with resected lung adenocarcinomas that harbor EGFR mutations. J Thorac Oncol. 2011 Mar;6(3):569-75.

27 Cheng H, Li XJ, Wang XJ, Chen ZW, Wang RQ, Zhong HC, et al. A meta-analysis of adjuvant EGFR-TKIs for patients with resected non-small cell lung cancer. Lung Cancer. 2019 Nov;137:7-13.

28 Yuan Y, Huang Q, Gu C, Chen H. Disease-free survival improved by use of adjuvant EGFR tyrosine kinase inhibitors in resectable nonsmall cell lung cancer: an updated meta-analysis. J Thorac Dis. 2017 Dec;9(12):5314-21.

29 Thatcher N, Chang A, Parikh P, Rodrigues Pereira J, Ciuleanu T, von Pawel J, et al. Gefitinib plus best supportive care in previously treated patients with refractory advanced nonsmall-cell lung cancer: results from a randomised, placebo-controlled, multicentre study (Iressa Survival Evaluation in Lung Cancer). Lancet. 2005 Oct;366(9496):1527-37.

30 Soria JC, Ohe Y, Vansteenkiste J, Reungwetwattana $\mathrm{T}$, Chewaskulyong $\mathrm{B}$, Lee $\mathrm{KH}$, et al.; FLAURA Investigators. Osimertinib in Untreated EGFR-Mutated Advanced NonSmall-Cell Lung Cancer. N Engl J Med. 2018 Jan;378(2):113-25.

31 Wu YL, Herbst RS, Mann H, Rukazenkov Y, Marotti M, Tsuboi M. ADAURA: Phase III, Double-Blind, Randomized Study of Osimertinib versus Placebo in EGFR Mutation-Positive Early-Stage NSCLC after Complete Surgical Resection. Clin Lung Cancer. 2018 Jul; 19(4):e533-6.

32 Ding PN, Lord SJ, Gebski V, Links M, Bray V, Gralla RJ, et al. Risk of Treatment-Related Toxicities from EGFR Tyrosine Kinase Inhibitors: A Meta-Analysis of Clinical Trials of Gefitinib, Erlotinib, and Afatinib in Advanced EGFR-Mutated Non-Small Cell Lung Cancer. J Thorac Oncol. 2017 Apr;12(4):633-43.

33 Miyahara T, Sueoka-Aragane N, Iwanaga K, Ureshino N, Komiya K, Nakamura T, et al. Severity and predictive factors of adverse events in pemetrexed-containing chemotherapy for non-small cell lung cancer. Med Oncol. 2017 Nov;34(12):195

34 Tang W, Li X, Xie X, Sun X, Liu J, Zhang J, et al. EGFR inhibitors as adjuvant therapy for resected non-small cell lung cancer harboring EGFR mutations. Lung Cancer. 2019 Oct; 136:6-14. 